\title{
No evidence for changes in skeletal muscle mass or weight during first-line chemotherapy for metastatic colorectal cancer
}

Sami Antoun ${ }^{1 *}$, Mohamed Amine Bayar ${ }^{2,3}$, Valérie Dyevre $^{2}$, Emilie Lanoy $^{2}$, Cristina Smolenschi $^{4}$ and Michel Ducreux ${ }^{4}$

\begin{abstract}
Background: Studies over the past 10 years strongly support an association between skeletal muscle mass (SMM) depletion and outcome in metastatic colorectal cancer (mCRC). Factors influencing SMM changes over time are, however, poorly studied. We analyzed the impact of SMM on overall survival and chemotherapy toxicities in mCRC patients treated with first-line chemotherapy. Changes in weight and body composition were evaluated during follow-up.
\end{abstract}

Methods: Patients enrolled in the randomized phase II ACCORD trial comparing two chemotherapy regimens were screened. Body composition parameters (SMM, adipose tissue) were assessed prospectively with computed tomography (CT) imaging, and toxicities were recorded. Mixed models were used to assess weight and BC changes during 4 months of treatment follow-up.

Results: Among 145 patients included in ACCORD, 76 had available baseline $C T$ scans and were included in the current study. Mean age was $60.6 \pm 10.0$ years, $50 \%$ were women, $82 \%$ had colon cancer, and $62 \%$ had two or more metastatic sites. At baseline, $49 \%$ had lost at least $5 \%$ of their initial weight, including $26 \%$ who had lost more than 10\%; $53 \%$ had SMM depletion. In this homogenous cohort, there were no statistically significant associations between SMM depletion and overall survival, progression-free survival or chemotherapy toxicity. There were no decreases in weight or SMM during follow-up. Weight and SMM changes were not influenced by diarrhea either grade 3-4 or any grade (reported in 74\% of patients). For patients with weight loss $\geq 10 \%$ at baseline, SMM increased significantly after 4 months of follow-up and after disease stabilization following chemotherapy $(P=0.008)$.

Conclusions: In a homogenous mCRC cohort, SMM depletion was not associated with survival or chemotherapy toxicity. Despite most patient experiencing diarrhea, no changes in weight or SMM were found during 4 months of follow-up. However, hypotheses deriving from our exploratory study have to be tested in further larger sample size studies.

Trial registration: Clinicaltrials.gov NCT00423696 (2011).

Keywords: Muscle mass depletion, Metastatic colorectal cancer, Weight loss, Body composition, Overall survival, Chemotherapy toxicity

\footnotetext{
* Correspondence: sami.antoun@gustaveroussy.fr

${ }^{1}$ Medical Emergency Unit in Oncology, Gustave Roussy Cancer Campus,

94800 Villejuif, France

Full list of author information is available at the end of the article
}

(c) The Author(s). 2019 Open Access This article is distributed under the terms of the Creative Commons Attribution 4.0 International License (http://creativecommons.org/licenses/by/4.0/), which permits unrestricted use, distribution, and reproduction in any medium, provided you give appropriate credit to the original author(s) and the source, provide a link to the Creative Commons license, and indicate if changes were made. The Creative Commons Public Domain Dedication waiver (http://creativecommons.org/publicdomain/zero/1.0/) applies to the data made available in this article, unless otherwise stated. 


\section{Background}

The prognosis of colorectal cancer (CRC), the third most frequent cancer in the Western world has changed dramatically with the advent of treatments combining cytotoxic drugs, targeted therapies, radiotherapy, and surgical resection of metastases [1]. Median survival in patients with metastatic CRC (mCRC) now exceeds 24 months $[2,3]$. Several multidimensional prognostic and predictive factors related to tumor responsiveness to therapies, pathological stage, and the patient's resistance capacity against the disease, are associated with outcome [4]. Among patient-related factors, nutritional parameters can influence outcomes in cancer patients [5].

In the past 10 years, studies have strongly suggested that skeletal muscle mass (SMM) depletion is associated with poorer survival outcomes independent of weight loss (WL) [6]. In CRC, the deleterious effect of SMM depletion on overall survival (OS) has been described for stage I to III disease [7], for patients undergoing adjuvant chemotherapy [8], as well as for short-term postoperative outcomes [9-12]. SMM depletion has also been associated with chemotherapy toxicity and discontinuation of chemotherapy treatments in many cancer types [13-15], and especially for CRC [8, 16-18].

As with most common solid tumors, CRC disease progression is associated with a progressive nutritional decline $[5,19]$. Loss of SMM and adipose tissue may be associated with the intense catabolism linked to progressive disease; in CRC, SMM loss depends on tumor stage and tumor evolution [20] and is accelerated at the end of life [10, 21]. The role of intrinsic factors (patient characteristics, nutritional status, and non-tumoral aspects) and treatment exposure, which could be involved in SMM changes over time, has been poorly studied [22, 23].

Most of the described associations between SMM depletion and outcomes, and most studies on weight and body composition (BC) parameter changes have several limitations including heterogeneity in terms of CRC stages, chemotherapy regimens, and treatment combinations (surgery, radiotherapy). In this study, we selected a homogenous population, in which all patients had the same stage of $\mathrm{mCRC}$, were receiving first-line chemotherapy, and were recruited in the context of a randomized clinical trial evaluating two chemotherapy regimens differentiated only by the modality of administration. Our objective was to analyze the impact of SMM and adipose tissue on OS, progressive-free survival (PFS), as well as on chemotherapy toxicities. The secondary objective was to analyze weight and body composition changes in a homogenous cohort of mCRC patients receiving first-line chemotherapy during the first 4 months after treatment initiation as well as the impact of tumor and patient characteristics on these changes.

\section{Methods}

\section{Patients}

Patients who were enrolled in the ACCORD trial between March 2006 and January 2008 at 15 centers in France, were screened. The ACCORD trial was a prospective multicenter, randomized, open-labelled, noncomparative phase II trial [2]. The aim of the ACCORD study was to evaluate the efficacy and safety of bevacizumab in combination with either oral capecitabine plus irinotecan (XELIRI) or 5-fluorouracil /leucovorin plus irinotecan (FOLFIRI) as first-line therapy for mCRC. To be eligible in the current study, patients had to have available computed tomography (CT) scans at baseline, 2 and 4 months, be aged $18-75$ years, with unresectable, histologically proven, measurable mCRC.

The ACCORD study was approved by ethics committee of the Kremlin-Bicêtre hospital and all patients provided informed consent. The additional analyses of clinical data and interpretation of body composition from CT images in the current study were approved by the Gustave Roussy independent ethics committees.

\section{CT image analysis of anthropometry and body composition}

According to the original study protocol, physical examination, and routine blood/urine analysis were assessed within 8 days before starting study treatment and were repeated every treatment cycle. Tumor assessments with abdominal CTs or magnetic resonance imaging were performed at baseline and every 8 weeks until progression. The same scanning techniques were used at each assessment. Weight and height were measured at baseline. For the current study, nutritional follow-up (weight and $\mathrm{BC}$ parameter changes) were assessed until the fourth month of treatment. Body mass index (BMI) was calculated (i.e., BMI $=$ weight $(\mathrm{kg}) /$ height $^{2}\left(\mathrm{~m}^{2}\right)$ ).

$\mathrm{BC}$ parameters were evaluated using the same CT images obtained for tumor assessment. All CT measurements were performed by the same operator who was blinded to patient information, clinical treatment and outcome. Measured variables were lumbar cross-sectional areas $\left(\mathrm{cm}^{2}\right)$ of skeletal muscle mass (SMM), visceral adipose tissue (VAT), and subcutaneous adipose tissue (SAT), as described previously [22, 24]. The third lumbar vertebra (L3) was chosen as the reference point $[25,26]$. CT images were analyzed using Slice-O-Matic software V4.3 (Tomovision, Montreal, Canada). These values were normalized for height scale and expressed in $\mathrm{cm}^{2} / \mathrm{m}^{2}$. To evaluate skeletal muscle density (SMD), we measured the mean radiation attenuation of skeletal muscle, which describes the input images read using Slice-O-Matic software. The pixel values of these images displayed in shades of grey as a correlate of muscle density represent the physical properties of the scanned 
tissue expressed in a numerical form by the mean Hounsfield Unit [27]. SMM depletion was defined according to the SMM index thresholds described by Martin et al. [6] (for women $\mathrm{SMM}<41 \mathrm{~cm}^{2} / \mathrm{m}^{2}$, for men $\mathrm{SMM}<43 \mathrm{~cm}^{2} / \mathrm{m}^{2}$ if BMI $<25 \mathrm{~kg} / \mathrm{m}^{2}$ and $<53 \mathrm{~cm}^{2} / \mathrm{m}^{2}$ if BMI $>25 \mathrm{~kg} / \mathrm{m}^{2}$ ).

\section{Endpoints}

According to the original study protocol, OS was defined as time from randomization in the ACCORD study to death or last follow-up. Progression was assessed based on investigators' tumor assessments. PFS was defined as time from randomization to progression or death not related to progression or last follow-up. Chemotherapy toxicity was evaluated using National Cancer Institute Common Terminology Criteria for Adverse Events, v3.0.

\section{Statistical analysis}

Continuous variables were summarized with the mean and the standard deviation, and the difference according to gender was tested using a Student's t-test or MannWhitney $\mathrm{U}$ test depending on normality. Categorical variables were summarized with the number of patients and frequency, and the difference according to gender was tested using a Pearson's chi-squared test or Fisher exact test depending on the expected numbers. The median follow-up was estimated using the reverse Kaplan-Meier method [28]. Survival was estimated using the KaplanMeier product limit method. For OS and PFS, estimates of hazard ratios (HRs) associated with BC parameters, Wald Chi-square $P$-values, and 95\% confidence intervals (CIs) were derived from Cox proportional hazard models. Effects of each body composition parameter-L3 skeletal muscle score, L3 visceral adipose tissue score, L3 subcutaneous adipose tissue score, L3 total adipose tissue score, Skeletal muscle density (HU) - on overall survival were derived from six separate multivariable model adjusted for age, sex, number of metastatic sites, treatment and BMI. Survival analyses were also done using Lasso penalized regressions as part of sensitivity analyses. Associations between $\mathrm{BC}$ parameters and global or specific toxicity occurrence were identified using a chi-squared test.

Mixed models were used to assess changes in $\mathrm{BC}$ parameters during the 4 months of follow-up, for patients with measurements at baseline (T0), 2 months (T2) and 4 months (T4). Linear mixed models were fitted for each BC index: weight, SMM, SMD, VAT and SAT. Full models were adjusted for age and tumor localization and included an interaction between gender and visit (T0, T2, T4). In the selected models, age, localization, and the interaction were only retained at level $p<.20$ in univariable model and according backward selection procedure. Gender was forced in mixed models for body composition, systematically. The correlation structure is a continuous AR (1), autocorrelation structure of order 1 , with a continuous time covariate. Only one random effect on the subject was included. For each model (weight, SMM, SMD, VAT and SAT), least square means and contrasts were reported with adjusted $P$-values using the Tukey method and the corresponding 95\% CI [29]. Weight loss and SMM depletion definitions refer to baseline. Analyses were performed using SAS Software, version 9.4 (SAS Institute, Cary, NC, USA) and given the sample size, they are considered explanatory.

\section{Results}

General, tumor, treatment and body composition characteristics

Among 145 patients included in the ACCORD, 76 met our inclusion criteria and were included in the survival analysis; 69 were excluded (Fig. 1). The mean age ( \pm standard deviation) was 60.6 years $( \pm 10.0)$, and 38 patients (50\%) were women (Table 1). Almost all patients (91\%) had ECOG PS 0-1, 62\% had two or more sites of metastasis and $82 \%$ had colon cancer. At baseline only $9 \%$ were considered malnourished according to the BMI $(<18.5)$, and $41 \%$ were overweight or obese (BMI $>25$ ). Among 65 patients with available data for their usual weight, 32 (49\%) had lost at least $5 \%$ of their initial weight at referral including 17 patients (26\%) who had lost $>10 \%$. Note that $53 \%$ of the population had SMM depletion according to Martin's thresholds. Fifty-seven patients were finally included in body composition changes analysis.

\section{Overall survival and progression-free survival}

For the 76 patients included, the median follow-up (Q1; Q3) was 21 months $(16 ; 29)$. At the cut-off date, 32 patients died and the median OS was 22 months (95\% CI: 20 - NR). In univariate analysis, OS was associated with age $(\mathrm{HR}=1.04 ; 95 \% \mathrm{CI}: 1.00-1.08 ; P=0.034)$, and SMM (HR $=0.65$; 95\% CI: $0.45-0.94 P=0.023$ ). In multivariable analysis, OS was not associated with changes in any BC parameters (SMM depletion, SMM score, VAT score, SAT score, TAT score or skeletal muscle density) (Table 2).

A total of 63 patients progressed and the median PFS was 9 months (95\% CI: 9-11). In univariate analysis, PFS was associated with age $(\mathrm{HR}=1.03$; $95 \% \mathrm{CI}: 1.00-1.06$; $P=0.020)$, and with SMD ( $\mathrm{HR}=0.97 ; 95 \% \mathrm{CI}$ : $0.95-$ 1.00]; $P=0.048$ ). Note that PFS was not associated with any $\mathrm{BC}$ parameters and the association with SMD was no longer significant (HR $=1.00$; $95 \%$ CI: $0.96-1.0,4 P=$ 0.97) (Additional file 1: Table S1).

\section{Chemotherapy toxicity}

Grade 3-4 chemotherapy-related toxicities occurred in 47 (62\%) patients. During the first 4 months of 


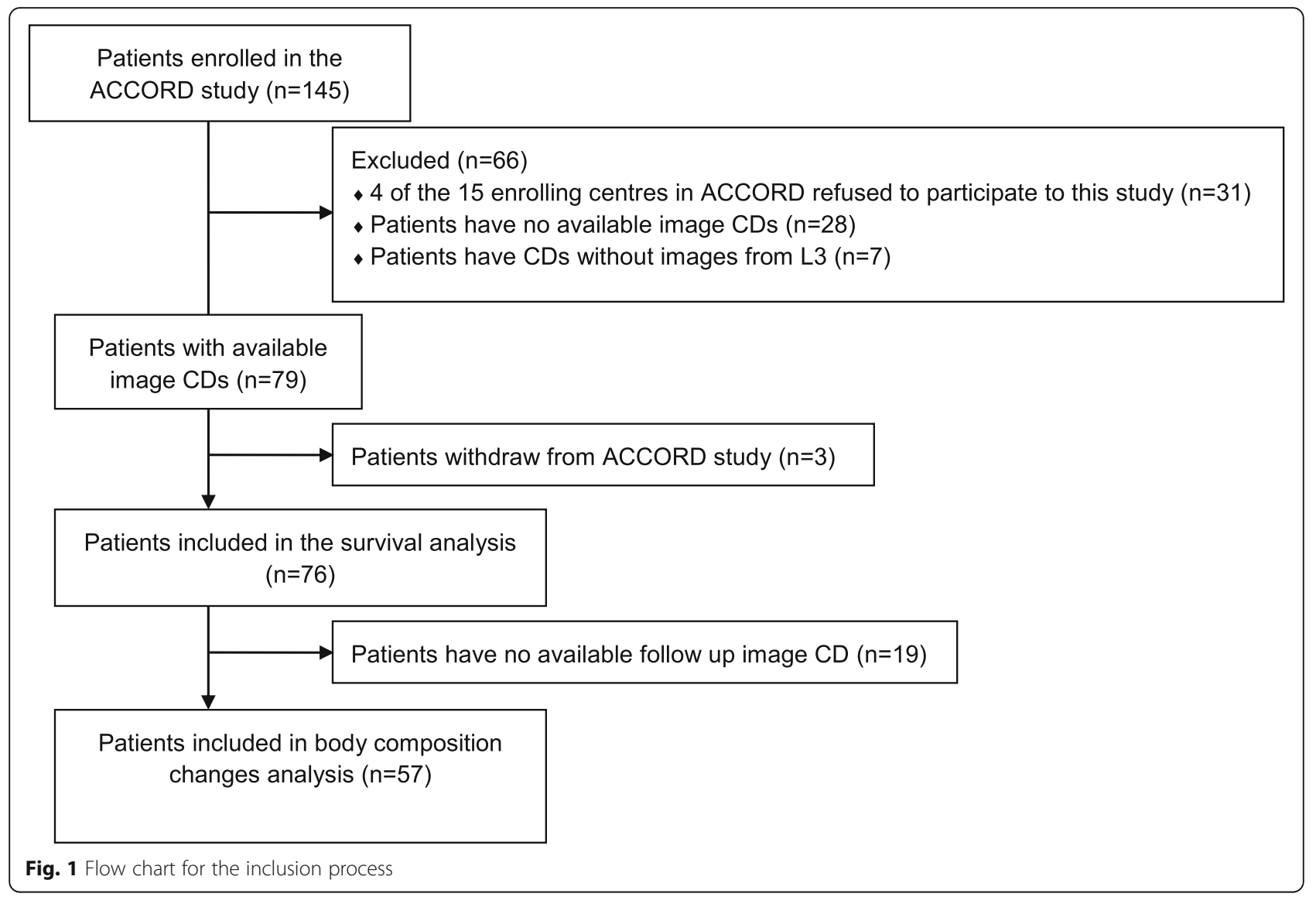

treatment, grade 3-4 neutropenia and diarrhea were observed in $22 \%$ and $14 \%$ of the patients respectively and any grade diarrhea were observed in $74 \%$ of the patients. There was no difference between patients with SMM depletion and those without SMM depletion in terms of incidence of any of the chemotherapy toxicities or for toxicities requiring changes to the regimen, irrespective of relationship to XELIRI, FOLFIRI or bevacizumab (Additional file 1: Table S2).

\section{Changes in body composition parameters and body weight during follow-up}

Of the 76 patients included in this study, 57 had available CT scans for both the 2 and 4-month follow-up. No decreases were seen in weight, SMM, or VAT during the 4 months of follow-up (Fig. 2, Table 3 and Additional file 1: Table S3). SMD and SAT decreased in women but not men, after 4 months of follow-up, mainly between 2 and 4 months for SMD 6.8 HU $(P=0.012)$, and over the 4 -month period for SAT $15.4 \mathrm{~cm}^{2} / \mathrm{m}^{2} \quad(P=0.03)$. The proportion of patients with SMM depletion decreased from $52.4 \%$ at baseline to $47.3 \%$ after 4 months of treatment.

SMM changes were not influenced by gender, age, initial values of adipose SMM, VAT or SAT, and were not dependent on the presence of SMM loss at baseline (assessed using mixed models). Weight and SMM changes were not influenced by grade 3-4 or any grade of diarrhea. For patients presenting with WL $>10 \%$ at baseline, SMM increased significantly after 4 months of follow-up $(P=0.008)$ (Fig. 3).

\section{Discussion}

This study in patients undergoing first-line treatment for mCRC demonstrated a number of noteworthy findings relevant to the role of SMM depletion in cancer patients. The first key observation is that no statistically significant or clinical relevant association was found between low SMM and adverse clinical outcomes. Secondly no changes were observed in weight, SMM, muscle density or adipose tissues during the 4-month follow-up.

Although some studies have described an association between SMM depletion before treatment and OS, we did not observe this association. Analyses in two large CRC cohorts, including 2407 and 804 patients, reported that SMM depletion was an independent prognostic factor for OS $[7,9]$. In contrast, McSorely et al. were unable to demonstrate a significant change in OS in CRC patients with SMM depletion [30]. The patients in these three studies presented similar disease characteristics; all 
Table 1 Patient, tumor, treatment and body composition characteristics at inclusion

\begin{tabular}{|c|c|c|c|c|}
\hline & Male $n=38$ (50\%) & Female $n=38(50 \%)$ & Total $n=76$ & $P$-value \\
\hline Age (years) & $60.7 \pm 9.1$ & $60.5 \pm 11.0$ & $60.6 \pm 10.0$ & 0.971 \\
\hline ECOG-PS & & & & 1.000 \\
\hline $0-1$ & $35(92 \%)$ & $34(90 \%)$ & 69 (91\%) & \\
\hline 2 & $3(8 \%)$ & $4(10 \%)$ & 7 (9\%) & \\
\hline Tumor type & & & & 1.000 \\
\hline colon & $31(82 \%)$ & $31(82 \%)$ & $62(82 \%)$ & \\
\hline rectum & $7(18 \%)$ & $7(18 \%)$ & $14(18 \%)$ & \\
\hline Number of metastatic sites & & & & 0.479 \\
\hline 1 & $13(34 \%)$ & $16(42 \%)$ & $29(38 \%)$ & \\
\hline$\geq 2$ & $25(66 \%)$ & $22(58 \%)$ & $47(62 \%)$ & \\
\hline Treatment regimen & & & & 0.163 \\
\hline XELIRI & $19(50 \%)$ & $13(34 \%)$ & $32(42 \%)$ & \\
\hline FOLFIRI & $19(50 \%)$ & $25(66 \%)$ & $44(58 \%)$ & \\
\hline Weight (kg) & $76.4 \pm 12.3$ & $57.7 \pm 10.8$ & $67.0 \pm 14.8$ & $<0.001$ \\
\hline Weight loss (WL) (\%) & $6.0 \pm 6.0$ & $6.0 \pm 7.9$ & $6.0 \pm 7.0$ & 0.564 \\
\hline WL category $5 \%$ cut-off & & & & 0.386 \\
\hline$\leq 5 \%$ & $19(58 \%)$ & $14(44 \%)$ & $33(51 \%)$ & \\
\hline$>5 \%$ & $14(42 \%)$ & $18(56 \%)$ & $32(49 \%)$ & \\
\hline Missing & $5(-)$ & $6(-)$ & $11(-)$ & \\
\hline WL category $10 \%$ cut-off & & & & 0.941 \\
\hline$\leq 10 \%$ & $25(76 \%)$ & $23(72 \%)$ & $48(74 \%)$ & \\
\hline$>10 \%$ & $8(24 \%)$ & $9(28 \%)$ & $17(26 \%)$ & \\
\hline Missing & $5(-)$ & $6(-)$ & $11(-)$ & \\
\hline BMI $\left(\mathrm{kg} / \mathrm{m}^{2}\right)$ & $25.6 \pm 3.8$ & $22.3 \pm 4.1$ & $23.9 \pm 4.2$ & $<0.001$ \\
\hline BMI category $\left(\mathrm{kg} / \mathrm{m}^{2}\right)$ & & & & 0.001 \\
\hline$<18.5$ & $0(0 \%)$ & $7(18 \%)$ & 7 (9\%) & \\
\hline $18.5-24.9$ & $16(42 \%)$ & $22(58 \%)$ & $38(50 \%)$ & \\
\hline $25-29.9$ & $15(40 \%)$ & $8(21 \%)$ & $23(30 \%)$ & \\
\hline$\geq 30$ & $7(18 \%)$ & $1(3 \%)$ & $8(11 \%)$ & \\
\hline SMM index $\left(\mathrm{cm}^{2} / \mathrm{m}^{2}\right)$ & $52.9 \pm 9.9$ & $37.9 \pm 5.4$ & $45.4 \pm 10.9$ & $<0.001$ \\
\hline $\mathrm{SMD}(\mathrm{HU})$ & $40.4 \pm 8.9$ & $40.0 \pm 11.2$ & $40.2 \pm 10.0$ & 0.881 \\
\hline VAT index $\left(\mathrm{cm}^{2} / \mathrm{m}^{2}\right)$ & $46.5 \pm 28.0$ & $23.8 \pm 20.5$ & $35.1 \pm 26.9$ & $<0.001$ \\
\hline SAT index $\left(\mathrm{cm}^{2} / \mathrm{m}^{2}\right)$ & $45.6 \pm 17.5$ & $54.2 \pm 30.5$ & $50.0 \pm 25.2$ & 0.285 \\
\hline SMM depletion & $12(32 \%)$ & $28(74 \%)$ & $40(53 \%)$ & $<0.001$ \\
\hline
\end{tabular}

Continuous variables are summarized with mean \pm standard deviation and the difference according to gender is tested using Wilcoxon-Mann-Whitney test. Categorical variables were summarized with the number of patients and the frequency, $\mathrm{n}(\%)$, and the difference according to gender was tested using a Pearson's chi-squared test or Fisher exact test when necessary

ECOG PS Eastern Cooperative Oncology Group Performance Status, XELIRI bevacizumab + oral capecitabine + irinotecan, FOLFIRI bevacizumab

+5 -fluorouracil/leucovorin (5FU/LV) + irinotecan

Weight loss $(\mathrm{WL})=$ (usual weight-weight at inclusion) / usual weight $\times 100$; usual weight was available for 65 patients. BMI Body Mass Index (weight (kg) / height ${ }^{2}$ $\left(\mathrm{m}^{2}\right)$ ), SMM skeletal muscle mass, SMD skeletal muscle density, HU Hounsfield Units, VAT visceral adipose tissue, SAT subcutaneous adipose tissue

SMM loss thresholds were those defined by Martin et al. [6]

had stage I-III CRC, underwent surgical resection and had an abdominal CT scan before surgery. A recent review analyzing contemporary studies concluded that patients with SMM depletion and low SMD are consistently associated with worse survival outcomes [31].
Nonetheless, most of the studies of this systematic review included resectable CRC or cohorts including both respiratory to gastrointestinal cancer patients. The authors also noted that significant methodological heterogeneity, mainly the lack of consensus for cut-offs 
Table 2 Univariate and multivariable analyses for overall survival

\begin{tabular}{|c|c|c|c|c|c|c|}
\hline \multirow[t]{2}{*}{ Characteristic } & \multirow[b]{2}{*}{ No. evt/ No. pts } & \multirow[b]{2}{*}{$\begin{array}{l}\text { Median OS } \\
(95 \% \mathrm{Cl})\end{array}$} & \multicolumn{2}{|c|}{ Univariate analysis } & \multicolumn{2}{|c|}{ Multivariable $^{a}$ analysis } \\
\hline & & & $\mathrm{HR}[95 \% \mathrm{Cl}]$ & $P$-value & $\mathrm{HR}[95 \% \mathrm{Cl}]$ & $P$-value \\
\hline Age (years) & & & $1.04[1.00 ; 1.08]$ & 0.034 & & \\
\hline \multicolumn{7}{|l|}{ Sex } \\
\hline Male & $17 / 37$ & $22(20 ; N R)$ & 1 & 0.900 & & \\
\hline Female & $15 / 38$ & $21(20 ; N R$ & $1.05[0.52 ; 2.1]$ & & & \\
\hline \multicolumn{7}{|l|}{ ECOG Performance status } \\
\hline $0-1$ & $32 / 68$ & $21(20 ; 24)$ & NA & NA & & \\
\hline 2 & $0 / 7$ & NA & NA & & & \\
\hline \multicolumn{7}{|l|}{ Number of metastatic sites } \\
\hline 1 & $11 / 28$ & $22(20 ; N A)$ & 1 & 0.500 & & \\
\hline$\geq 2$ & $21 / 47$ & $22(19 ; \mathrm{NA})$ & $1.29[0.62 ; 2.68]$ & & & \\
\hline \multicolumn{7}{|l|}{ Treatment } \\
\hline XELIRI & $11 / 31$ & $22(20 ; N A)$ & 1 & 0.150 & & \\
\hline FOLFIRI & $21 / 44$ & $21(20 ; 24)$ & $1.71[0.82 ; 3.56]$ & & & \\
\hline \multicolumn{7}{|l|}{ Body mass index category $\left(\mathrm{kg} / \mathrm{m}^{2}\right)$} \\
\hline$<25$ & $22 / 45$ & $21(19 ; N A)$ & 1 & 0.180 & & \\
\hline$\geq 25$ & $10 / 30$ & $24(21 ; N A)$ & $0.60[0.28 ; 1.26]$ & & & \\
\hline \multicolumn{7}{|l|}{ Model 1: SMM depletion at baseline } \\
\hline No & $13 / 35$ & $24(20 ; N A)$ & 1 & 0.150 & 1 & 0.791 \\
\hline Yes & $19 / 40$ & $21(20 ; 23)$ & $1.69[0.83 ; 3.43]$ & & $1.14[0.44 ; 2.98]$ & \\
\hline Model 2: L3 skeletal muscle score & & & $0.65[0.45 ; 0.94]$ & 0.023 & $0.71[0.41 ; 1.22]$ & 0.211 \\
\hline Model 3: L3 visceral adipose tissue score & & & $1.03[0.71 ; 1.48]$ & 0.880 & $1.05[0.67 ; 1.64]$ & 0.833 \\
\hline Model 4: L3 subcutaneous adipose tissue score & & & $0.68[0.46 ; 1.01]$ & 0.054 & $0.71[0.44 ; 1.14]$ & 0.153 \\
\hline Model 5: L3 total adipose tissue score & & & $0.84[0.57 ; 1.23]$ & 0.370 & $0.87[0.53 ; 1.38]$ & 0.557 \\
\hline Model 6: Skeletal muscle density (HU) & & & $0.98[0.94 ; 1.01]$ & 0.210 & $1.01[0.95 ; 1.06]$ & 0.868 \\
\hline
\end{tabular}

No. evt number events, No. pts. number patients, HR Hazard ratio, Cl confidence interval, $p$-value, ECOG Eastern Cooperative Oncology Group, HU Hounsfield Units, NA not available, SMM Skeletal Muscle mass depletion thresholds were those defined by Martin et al. [6]

${ }^{a}$ Each 6 multivariable models assessing effect of each body component parameter on overall survival were adjusted for age, sex, number of metastases, treatment, BMI

defining SMM depletion, limited the strength of their conclusions [31]. The only study with a cohort of mCRC similar to ours did not observe an association between SMM depletion at inclusion and OS [18]. However, in contrast to our study, Blauwhoff-Buskerwole et al. observed that SMM loss during follow-up was independently associated with survival (HR 4.47; $P<0.001$ ). Differences in anticancer treatments, could potentially explain these discordances. In their study, $22 \%$ of the population received a reduced chemotherapy initial dose and only $23 \%$ (compared to $88 \%$ in our study), received a second-line of chemotherapy, suggesting their population may have had more aggressive disease, or a worse prognosis at inclusion.

It is interesting to note that the first study showing the relationship between SMM depletion and chemotherapy toxicity was reported in CRC patients [32]. This pioneering article was followed by others with similar results $[8,16,17]$. The recent C-SCANS study in a large population $(N=533)$ showed that patients with the lowest tertile of muscle mass were more likely to experience hematologic toxicity and early treatment discontinuation than those in the highest [18]. We however did not identify a relationship between SMM depletion and chemotherapy toxicity but our study sample size did not allow us to adjust for confounding factors. These discrepancies could be linked to our small sample size and/or to different methodology; we analyzed low muscle mass by dichotomous variable (SMM depletion according to Martin's cut off compared to patients without SMM depletion) rather than with tertiles. The two studies also differ in terms of chemotherapy regimens, with different rates of hematologic toxicity (44\% neutropenia in the CSCANS study compared to $22 \%$ here). The abovementioned studies [8, 16-18] present methodological 

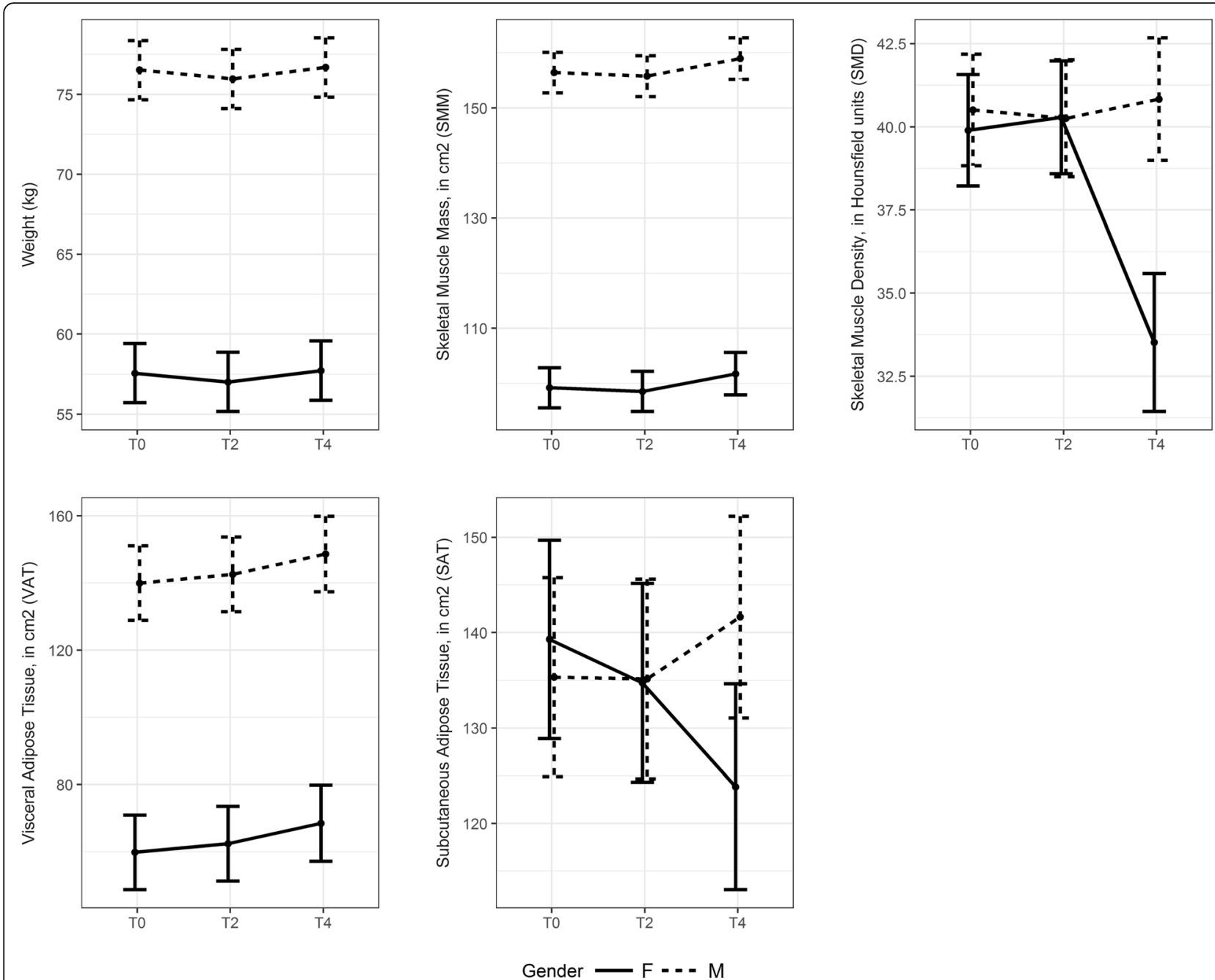

Fig. 2 Estimate changes from first CT-scan evaluation. Interaction plot for least square means \pm SD for women (solid lines) and men (dotted lines), for weight $(\mathrm{kg})$ between T0-T4 $P=0.935$, for skeletal muscle mass area (SMM) $\left(\mathrm{cm}^{2}\right)$ between T0-T4 $P=0.544$, for skeletal muscle density (SMD) (Hounsfield unit) between T0-T4 for women $P=0.038$ for men $P=0.990$, for visceral adipose tissue area $\left(\mathrm{cm}^{2}\right)$ between T0-T4 $P=0.161$, for visceral adipose tissue area $\left(\mathrm{cm}^{2}\right)$ between T0-T4 for women $P=0.030$ for men $P=0.500$

heterogeneities that limit the possibilities of comparison. The main hurdle is the absence of a defined threshold for SMM depletion and variations in the choice of muscle surfaces used to measure SMM. It would be interesting to repeat the analyses in the CSCANS study [18] using the cut-off values of SMM depletion used in other studies $[6,9]$. This lack of reproducibility could explain why, 10 years after the first publication, SMM is not routinely used for scaling chemotherapy doses.

For women, a decrease in muscle density and SAT was observed after 2 months of follow-up. Decreased SMD could be associated with increased lipid droplet deposit [27]. Stephen et al. carried out a quantitative morphological examination of lipid droplets in muscle biopsies of gastrointestinal cancer patients [33]. Significantly more intramyocellular lipid droplets were seen in patients with lower VAT measures with a trend toward an association between droplet number and SAT. The authors suggest that an increase in lipolysis is responsible for the decreased adipose tissue and an increased deposition of lipids within muscle (low SMD), probably reflecting an imbalance between fatty acid supply and use by muscle. This may explain our results observed in women. Difference in adipose tissue distribution and metabolism according to sex [34] could explain the significant results we observed in women and not in men. We should focus on intrinsic factors such as gender when studying SMM and adipose tissue changes during $\mathrm{mCRC}$ treatment and follow-up, as reported by Malietzis et al. [20].

Decreased weight and SMM in cancer patients could be a consequence of reduced dietary intake and/or metabolic changes linked to the cancer or its treatment $(49 \%$ 
Table 3 Changes in body weight, tissue areas and muscle density during 4 months of follow-up, by gender. Fifty-seven patients were included in body composition changes analysis

\begin{tabular}{lllll}
\hline & Visit & LS Means $[95 \% \mathrm{Cl}]$ & LS Means [95\% Cl] - Women & LS Means [95\% CI] - Men \\
\hline Weight $(\mathrm{kg})$ & T0 & $67.04[64.42 ; 69.66]$ & $57.57[53.89 .61 .25]$ & $76.51[72.83 .80 .19]$ \\
& T2 & $66.49[63.86 ; 69.11]$ & $57.01[53.33 .60 .70]$ & $75.96[72.28 .79 .64]$ \\
SMM $\left(\mathrm{cm}^{2}\right)$ & T4 & $67.20[64.57,69.84]$ & $57.73[54.04 .61 .42]$ & $76.68[72.99 .80 .37]$ \\
& T0 & $127.83[122.47 ; 133.19]$ & $99.23[91.96 ; 106.51]$ & $156.42[149.14 ; 163.70]$ \\
& T2 & $127.17[121.74 ; 132.59]$ & $98.57[91.26 ; 105.89]$ & $155.76[148.43 ; 163.10]$ \\
SMD $(\mathrm{HU})$ & T4 & $130.36[124.60 ; 136.12]$ & $101.76[94.11 ; 109.42]$ & $158.95[151.45 ; 166.45]$ \\
& T0 & $40.20[37.84 ; 42.56]$ & $39.90[36.56 ; 43.23]$ & $40.51[37.17 ; 43.85]$ \\
& T2 & $40.27[37.84 ; 42.71]$ & $40.29[36.91 ; 43.67]$ & $40.26[36.76 ; 43.76]$ \\
VAT $\left(\mathrm{cm}^{2}\right)$ & T4 & $37.18[34.41 ; 39.94]$ & $33.52[29.38 ; 37.65]^{\mathrm{a}}$ & $40.86[37.16 ; 44.51]$ \\
& T0 & $99.86[83.92 ; 115.80]$ & $59.77[37.58 ; 81.96]$ & $139.95[117.76 ; 162.14]$ \\
& T2 & $102.48[86.48 ; 118.47]$ & $62.39[40.16 ; 84.61]$ & $142.57[120.33 ; 164.80]$ \\
SAT $\left(\mathrm{cm}^{2}\right)$ & T4 & $108.56[92.15 ; 124.96]$ & $68.46[45.84 ; 91.09]$ & $148.65[126.22 ; 171.07]$ \\
& T0 & $137.32[122.65 ; 152.00]$ & $139.30[118.57 ; 160.03]$ & $135.34[114.57 ; 156.11]$ \\
& T2 & $134.95[120.23 ; 149.67]$ & $134.74[113.98 ; 155.51]$ & $135.15[114.29 ; 156.01]$ \\
& T4 & $132.75[117.69 ; 147.80]$ & $123.85[102.34 ; 145.36]^{\mathrm{b}}$ & $141.64[120.58 ; 162.70]$ \\
\hline
\end{tabular}

For each body component, the fitted model contained only gender and visit

LS lean square means, Cl Confidence Interval, SMM skeletal muscle mass index $\left(\mathrm{cm}^{2}\right)$, SMD skeletal muscle density, HU Hounsfield Unit, VAT visceral adipose tissue $\left(\mathrm{cm}^{2}\right)$, SAT subcutaneous adipose tissue $\left(\mathrm{cm}^{2}\right)$

${ }^{\mathrm{a}} P=0.038 ;{ }^{\mathrm{b}} P=0.030$

of patients in our study had lost more than $5 \%$ of their usual weight at referral). The most interesting finding is the stabilization of weight and SMM after initiating treatment. Of note, all but one patient in our study had stable disease. This offers supplementary proof that

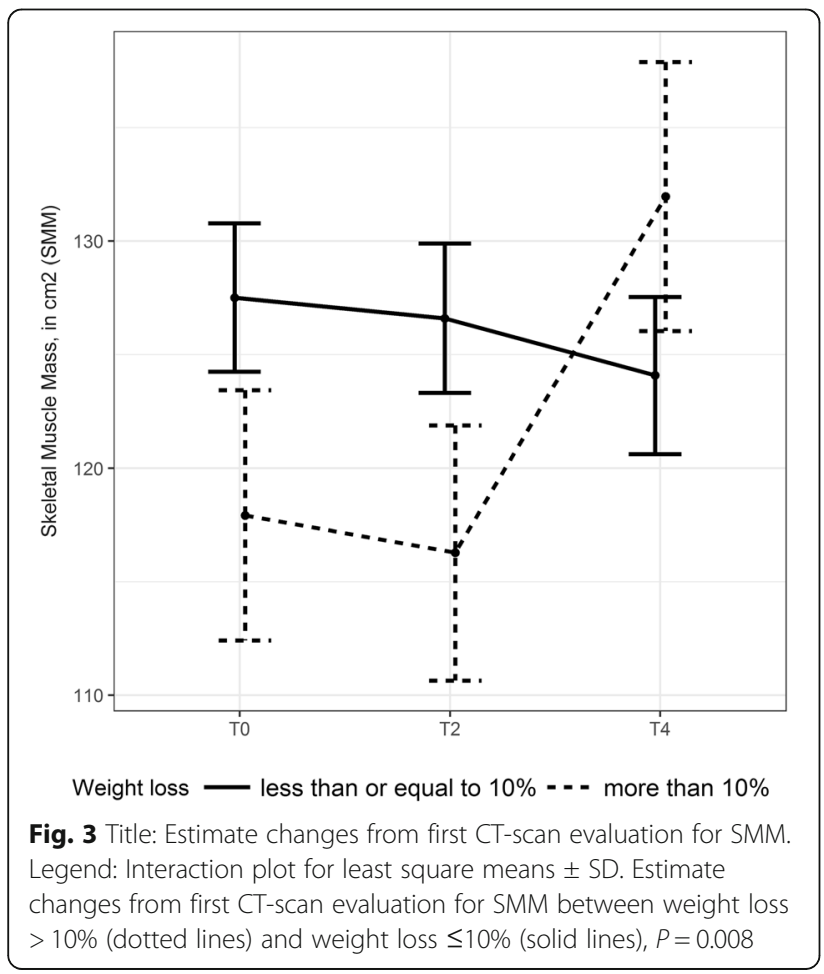

tumor evolution is the main factor impacting weight and SMM loss. Lieffers et al. have reported in mCRC that the most rapid decreases in SMM and adipose tissues were observed when tumor burden increased rapidly [10]. Others have questioned the role of anticancer treatment in SMM and WL. Poterucha et al. observed a decrease in SMM in mCRC patients and suggest that bevacizumab may be responsible [35]. The study by Blauwhoff-Buskerwole et al. showed a $6.1 \%$ decrease in SMM in a cohort similar to ours [19]. A recent publication described muscle mass changes during systemic treatment in a comparable, but larger group of 450 mCRC patients [36]. This study found that patients on average lost 0.7 (95\% CI $-1.11 ;-0.26) \mathrm{kg}$ SMM during the 4 months of first line intensive (carboplatine-oxaliplatinebevacizumab treatment CAPOX-B). Subsequently SMM recovered after subsequent less intensive carboplatineoxaliplatine or observation treatment, and again decreased during more intensive CAPOX-B or other reintroduction treatment [36]. From these two studies with the only apparent difference between our population and theirs being the chemotherapy regimens (irinotecan versus oxaliplatin respectively), we can hypothesize that the SMM decrease observed in the Blauwhoff-Buskerwole and Kurk studies is a direct effect of oxaliplatine, as described for other anti-cancer regiment: targeted therapies [22], doxorubicin [37, 38], and cisplatin [39]. This is not however supported by a study in mice showing that unlike oxaliplatin, irinotecan causes marked depletion in SMM [40]. 
Together, these findings highlight the importance of considering the toxic effects of chemotherapy on SMM, and could explain the observed discordant results.

In addition to metabolic abnormalities, another component relating to WL in cancer patients is decreased food intake. In 1131 hospitalized CRC patients, unintentional WL was associated with decreased intake [41]. In our population, all grade diarrheas was observed in $74 \%$ of cases, with grade 3-4 in $14 \%$. Despite these digestive and nutritional symptoms, which can decrease nutriment intake, we did not observe any consequences on weight or SMM, supporting the absence of nutritional disorders during early mCRC treatment despite gastrointestinal symptoms linked to chemotherapy toxicities.

WL and SMM depletion in mCRC patients during first-line anticancer chemotherapy seem not to be influenced by the initial nutritional status since no association was seen between the rate of SMM loss and WL for any nutritional parameters at inclusion, including BMI, SMM, muscle density, SMM depletion and adipose tissue. The only parameter associated with the rate of SMM loss is WL prior to inclusion, with significantly increased SMM during 4 months of follow-up in patients with WL $>10 \%$ at inclusion. We can hypothesize that SMM could be restored by treatments which control cancer evolution and that worse the metabolic abnormality causing muscle protein breakdown the greater this effect, reaching a critical level of WL $>10 \%$.

We are aware of several limitations of this study. Our study was designed to assess if previous findings about body composition are reproducible for different anticancer therapies; however, due to its small sample size and since consecutive CT images were available in only part of the patients, our study was exploratory exclusively. Of note, this small sample size induces a low power and prevented us from adjusting toxicity analyses for confounding factors. Another limitation is the delay between patient's inclusion and our study. It may be considered a long one not representing the current management and the current treatment of CRC in the time of molecular abnormality screening and targeted therapies.

\section{Conclusion}

In a homogenous cohort of mCRC patients treated with first-line chemotherapy, SMM depletion was not significantly associated with adverse clinical outcomes in terms of either survival or chemotherapy toxicities. These results differ from those observed by other groups, clearly highlighting the need for standardized definitions of SMM depletion thresholds and of the muscle surfaces to measure SMM. Limiting methodological heterogeneity will improve our understanding of the prognostic role of SMM depletion. The fact that we did not observe any changes in weight and SMM during the first 4 months of follow-up is surprising given the hypercatabolism observed in mCRC (half of the patients had WL before treatment), the high incidence of gastrointestinal toxicity, and the potential protein breakdown effect of chemotherapy. Our study also provides supplementary proof that tumor evolution is the main factor of weight and SMM loss, with stable disease, irrespective of nutritional and gastrointestinal disorders, maintaining weight and SMM. This highlights the important role of metabolic alterations and systemic inflammatory processes in muscle wasting. However, our results are not based on a large sample size, they did not indicate that SMM depletion was associated with adverse clinical outcomes, and further studies are necessary before drawing definitive conclusions.

\section{Additional file}

Additional file 1: Table S1. Univariate and multivariable analyses for progression-free survival. Table S2. Chemotherapy toxicities on treatment according to Skeletal Muscle Mass loss. Table S3. Rates of change in weight, tissue areas, and muscle density during 4 months of follow-up. (DOCX $48 \mathrm{~kb}$ )

\section{Abbreviations}

BC: Body composition; BMI: Body mass index; CT: Computed tomography; mCRC: Metastatic colorectal cancer; OS: Overall survival; SAT: Subcutaneous adipose tissue; SMD: Skeletal muscle radiodensity; SMM: Skeletal muscle mass; VAT: Visceral adipose tissue; WL: Weight loss

\section{Acknowledgements}

We are grateful to two reviewers for helpful comments that have helped us to strengthen the manuscript. The authors thank Sarah MacKenzie, PhD for editing.

\section{Authors' contributions}

SA, EL, CS and MD made substantial contributions to the conception and to the design of the work; MB, VD and EL conducted the statistical analyses; SA, and MD interpreted data and wrote the first draft of the manuscript. CS and MD substantively revised it. All authors have approved the submitted version and have agreed both to be personally accountable for the author's own contributions and to ensure that questions related to the accuracy or integrity of any part of the work, even ones in which the author was not personally involved, are appropriately investigated, resolved, and the resolution documented in the literature

\section{Funding}

This research did not receive any specific grant from any funding agency in the public, commercial or not-for-profit sector.

\section{Availability of data and materials}

The datasets used and/or analyzed in the current study are available from the corresponding author on reasonable request.

\section{Ethics approval and consent to participate}

Patients eligible in the current study were enrolled in the ACCORD trial, a prospective multicenter, randomized, open-labelled, non-comparative phase II trial [2]. The ACCORD study was approved by ethics committee of the Kremlin-Bicêtre hospital and all patients provided informed written consent (clinicaltrials.gov identifier NCT00423696). The additional analyses of clinical data and interpretation of body composition from CT images in the current study were approved by the Gustave Roussy independent ethics

committees. 


\section{Consent for publication}

Not applicable.

\section{Competing interests}

The authors declare that they have no competing interests.

\section{Author details}

'Medical Emergency Unit in Oncology, Gustave Roussy Cancer Campus, 94800 Villejuif, France. ${ }^{2}$ Department of Biostatics and Epidemiology, Gustave Roussy Cancer Campus, 94800 Villejuif, France. ${ }^{3}$ CESP, Faculté de Médecine, Université Paris Sud, INSERM, Université Paris Saclay, 94805 Villejuif, France. ${ }^{4}$ Department Medical Oncology, Gustave Roussy Cancer Campu, 94800 Villejuif, France.

Received: 15 December 2018 Accepted: 23 August 2019 Published online: 28 August 2019

\section{References}

1. Siegel R, Naishadham D, Jemal A. Cancer statistics. CA Cancer J Clin. 2013; 63:11-30.

2. Ducreux M, Adenis A, Pignon JP, et al. Efficacy and safety of bevacizumab -based combination regimens in patients with previously untreated metastatic colorectal cancer: final results from a randomised phase II study of bevacizumab plus 5-fluorouracil, leucovorin plus irinotecan versus bevacizumab plus capecitabine plus irinotecan (FNCLCC ACCORD 13/0503 study). Eur J Cancer. 2013:49:1236-45.

3. Ye LC, Liu TS, Ren L, et al. Randomized controlled trial of cetuximab plus chemotherapy for patients with KRAS wild-type unresectable colorectal liver-limited metastases. J Clin Oncol. 2013:31:1931-8.

4. De Divitiis C, Nasti G, Montano M, Fisichella R, laffaioli RV, Berretta M. Prognostic and predictive response factors in colorectal cancer patients: between hope and reality. World J Gastroenterol. 2014;20:15049-59.

5. Meyerhardt JA, Kroenke $\mathrm{CH}$, Prado $\mathrm{CM}$, et al. Association of weight change after colorectal cancer diagnosis and outcomes in the Kaiser Permanente northern California population. Cancer Epidemiol Biomark Prev. 2017;26:30-7.

6. Martin L, Birdsell L, Macdonald N, et al. Cancer cachexia in the age of obesity: skeletal muscle depletion is a powerful prognostic factor, independent of body mass index. J Clin Oncol. 2013;31:1539-47.

7. Cespedes Feliciano EMC, Kroenke CH, et al. Association of systemic inflammation and sarcopenia with survival in non-metastatic colorectal cancer: results from the C SCANS study. JAMA Oncol. 2017:3:e172319.

8. Jung HW, Kim JW, Kim JY, et al. Effect of muscle mass on toxicity and survival in patients with colon cancer undergoing adjuvant chemotherapy. Support Care Cancer. 2015;23:687-94.

9. Malietzis G, Currie AC, Athanasiou T, et al. Influence of body composition profile on outcomes following colorectal cancer surgery. $\mathrm{Br}$ J Surg. 2016;103:572-80

10. Lieffers JR, Mourtzakis M, Hall KD, McCargar $L$, Prado CM, Baracos VE. A viscerally driven cachexia syndrome in patients with advanced colorectal cancer: contributions of organ and tumor mass to whole-body energy demands. Am J Clin Nutr. 2009:89:1173-9.

11. Endo T, Momoki C, Yamaoka M, et al. Validation of skeletal muscle volume as a nutritional assessment in patients with gastric or colorectal cancer before radical surgery. J Clin Med Res. 2017:9:844-59.

12. Chemama S, Bayar MA, Lanoy E, et al. Sarcopenia is associated with chemotherapy toxicity in patients undergoing cytoreductive surgery with hyperthermic intraperitoneal chemotherapy for peritoneal carcinomatosis from colorectal cancer. Ann Surg Oncol. 2016:23:3891-8.

13. Antoun S, Borget I, Lanoy E. Impact of sarcopenia on the prognosis and treatment toxicities in patients diagnosed with cancer. Curr Opin Support Palliat Care. 2013;7:383-9.

14. Sjøblom B, Benth JŠ, Grønberg BH, et al. Drug dose per kilogram lean body mass predicts hematologic toxicity from carboplatin-doublet chemotherapy in advanced non-small-cell lung cancer. Clin Lung Cancer. 2017;18:e129-36.

15. Prado CM, Maia YL, Ormsbee M, Sawyer MB, Baracos VE. Assessment of nutritional status in cancer--the relationship between body composition and pharmacokinetics. Anti Cancer Agents Med Chem. 2013;13:1197-203.

16. Barret M, Antoun S, Dalban C, et al. Sarcopenia is linked to treatment toxicity in patients with metastatic colorectal cancer. Nutr Cancer. 2014;66:583-9.
17. Ali R, Baracos VE, Sawyer MB, et al. Lean body mass as an independent determinant of dose-limiting toxicity and neuropathy in patients with colon cancer treated with FOLFOX regimens. Cancer Med. 2016;5:607-16.

18. Cespedes Feliciano EM, Lee VS, et al. Muscle mass at the time of diagnosis of non-metastatic colon cancer and early discontinuation of chemotherapy, delays, and dose reductions on adjuvant FOLFOX: the C-SCANS study. Cancer. 2017;123:4868-77.

19. Blauwhoff-Buskermolen S, Versteeg KS, de van der Schueren MA, et al. Loss of muscle mass during chemotherapy is predictive for poor survival of patients with metastatic colorectal cancer. J Clin Oncol. 2016;34:1339-44.

20. Malietzis G, Currie AC, Johns N, et al. Skeletal muscle changes after elective colorectal cancer resection: a longitudinal study. Ann Surg Oncol. 2016:23:2539-47.

21. Prado CM, Sawyer MB, Ghosh S, et al. Central tenet of cancer cachexia therapy: do patients with advanced cancer have exploitable anabolic potential? Am J Clin Nutr. 2013;98:1012-9.

22. Antoun S, Birdsell L, Sawyer MB, Venner P, Escudier B, Baracos VE. Association of skeletal muscle wasting with treatment with sorafenib in patients with advanced renal cell carcinoma: results from a placebo -controlled study. J Clin Oncol. 2010;28:1054-60.

23. Atlan P, Bayar MA, Lanoy E, et al. Factors which modulate the rates of skeletal muscle mass loss in non-small cell lung cancer patients: a pilot study. Support Care Cancer. 2017;25:3365-73.

24. Prado CM, Lieffers JR, McCargar $L J$, et al. Prevalence and clinical implications of sarcopenic obesity in patients with solid tumours of the respiratory and gastrointestinal tracts: a population-based study. Lancet Oncol. 2008;9:629-35.

25. Shen W, Punyanitya M, Wang Z, et al. Total body skeletal muscle and adipose tissue volumes: estimation from a single abdominal cross-sectional image. J Appl Physiol (Bethesda, Md : 1985). 2004;9: 2333-8.

26. Mourtzakis M, Prado CM, Lieffers JR, Reiman T, McCargar LJ, Baracos VE. A practical and precise approach to quantification of body composition in cancer patients using computed tomography images acquired during routine care. Appl Physiol Nutr Metab. 2008;33:997-1006.

27. Goodpaster BH, Kelley DE, Thaete FL, He J, Ross R. Skeletal muscle attenuation determined by computed tomography is associated with skeletal muscle lipid content. J Appl Physiol (Bethesda, Md: 1985). 2000; 89:104-10.

28. Schemper M, Smith TL. A note on quantifying follow-up in studies of failure time. Control Clin Trials. 1996;17:343-6.

29. Bayar MA, Antoun S, Lanoy E. Statistical approaches for evaluating body composition markers in clinical cancer research. Expert Rev Anticancer Ther. 2017;17:311-8.

30. McSorley ST, Black DH, Horgan PG, McMillan DC. The relationship between tumour stage, systemic inflammation, body composition and survival in patients with colorectal cancer. Clin Nutr. 2018:37:1279-85.

31. Hopkins JJ, Skubleny D, Bigam DL, Baracos VE, Eurich DT, Sawyer MB. Barriers to the interpretation of body composition in colorectal Cancer: a review of the methodological inconsistency and complexity of the $\mathrm{CT}$ -defined body habitus. Ann Surg Oncol. 2018;25:1381-94.

32. Prado CM, Baracos VE, McCargar LJ, et al. Body composition as an independent determinant of 5-fluorouracil-based chemotherapy toxicity. Clin Cancer Res. 2007;13:3264-8.

33. Stephens NA, Skipworth RJ, Macdonald AJ, Greig CA, Ross JA, Fearon KC. Intramyocellular lipid droplets increase with progression of cachexia in cancer patients. J Cachexia Sarcopenia Muscle. 2011;2:111-7.

34. Lee M, Wu Y, Fried SK. Adipose tissue heterogeneity: implication of depot differences in adipose tissue for obesity complications. Mol Asp Med. 2013;34:1-11.

35. Poterucha T, Burnette $B$, Jatoi A. A decline in weight and attrition of muscle in colorectal cancer patients receiving chemotherapy with bevacizumab. Med Oncol. 2012:29:1005-9.

36. Kurk SA, Peeters PHM, Dorresteijn B, de Jong PA, Jourdan M, Kuijf HJ, Punt CJA, Koopman M, May AM. Impact of different palliative systemic treatments on skeletal muscle mass in metastatic colorectal cancer patients. J Cachexia Sarcopenia Muscle. 2018;9:909-19.

37. Gilliam LA, Ferreira LF, Bruton JD, et al. Doxorubicin acts through tumor necrosis factor receptor subtype 1 to cause dysfunction of murine skeletal muscle. J Appl Physiol (1985). 2009;107:1935-42.

38. van Norren K, van Helvoort A, Argilés JM, et al. Direct effects of doxorubicin on skeletal muscle contribute to fatigue. Br J Cancer. 2009;100:311-4. 
39. Hojman P, Fjelbye J, Zerahn B, et al. Voluntary exercise prevents cisplatin-induced muscle wasting during chemotherapy in mice. PLoS One. 2014;9:e109030.

40. Barreto R, Waning DL, Gao H, Liu Y, Zimmers TA, Bonetto A. Chemotherapy -related cachexia is associated with mitochondrial depletion and the activation of ERK1/2 and p38 MAPKs. Oncotarget. 2016;7:43442-60.

41. van der Werf A, Arthey K, Hiesmayr M, et al. The determinants of reduced dietary intake in hospitalised colorectal cancer patients. Support Care Cancer. 2018;26:2039-47.

\section{Publisher's Note}

Springer Nature remains neutral with regard to jurisdictional claims in published maps and institutional affiliations.

Ready to submit your research? Choose BMC and benefit from:

- fast, convenient online submission

- thorough peer review by experienced researchers in your field

- rapid publication on acceptance

- support for research data, including large and complex data types

- gold Open Access which fosters wider collaboration and increased citations

- maximum visibility for your research: over $100 \mathrm{M}$ website views per year

At $\mathrm{BMC}$, research is always in progress.

Learn more biomedcentral.com/submissions 